\title{
On Professor Whittaker's solution of differential equations by definite integrals: Part I
}

By W. O. Kermack (Royal College of Physicians, Edinburgh), and W. H. MaCrea (Edinburgh University).

(Received and read 16th January 1931).

§1. In the preceding paper Professor Whittaker has given a general method for the solution of differential equations by means of definite integrals. It depends on finding a solution $\chi(q, Q)$ of an auxiliary pair of simultaneous partial differential equations to be derived from an arbitrary contact transformation by changing the momentum variables into differential operators. The first object of the present paper is to arrive at a method for passing from the contact transformation in its algebraic form to these partial differential equations, in a manner which is unambiguous and which makes them compatible. We show too how to obtain any number of such pairs of equations from any given contact transformation. Successive transformations are also discussed.

$\S 2$. The derivation of the equations for $\chi$. Consider the contact transformation derived from the function ${ }^{1} W(q, Q)$ which transforms the variables $q, p$ into the variables $Q, P$ according to the equations

$$
\begin{aligned}
& P-\frac{\partial W}{\partial Q}=0, \\
& p+\frac{\partial W}{\partial q}=0 .
\end{aligned}
$$

Let us now regard $P, p$ as operators given by ${ }^{2}$

$$
P \equiv \frac{\partial}{\partial Q}, \quad p \equiv-\frac{\partial}{\partial q},
$$

1 The theory is given explicitly for a single pair of variables $q, p$ (or $Q, P$ ). It is clear, however, that it may be generalised at once to include any number of pairs.

2 Professor Whittaker takes both signs positive; but it is more convenient for the present method to take one positive and one negative. 
and consider the simultaneous equations for the function $\chi(Q, q)$,

that is

$$
\begin{aligned}
& {\left[P-\frac{\partial W}{\partial Q}\right] \chi=0} \\
& {\left[p+\frac{\partial W}{\partial q}\right] \chi=0,}
\end{aligned}
$$

$$
\begin{aligned}
& \frac{\partial \chi}{\partial Q}-\frac{\partial W}{\partial Q} \cdot x=0 \\
& \frac{\partial \chi}{\partial q}-\frac{\partial W}{\partial q} \cdot \chi=0 .
\end{aligned}
$$

These obviously possess the solution

$$
x=e^{m}
$$

It is not necessary to include an arbitrary multiplicative factor.

Now this $\chi$ will satisfy any equations derived from $\left(4^{\prime}\right),\left(5^{\prime}\right)$ by multiplying them by any functions of $q$ or $Q$, or by differentiating them with respect to $q$ or $Q$, or by any combination of such processes. In particular it will satisfy the equations

$$
\begin{aligned}
& \mathbf{Q}\left(q,-\frac{\partial}{\partial q}\right) \chi-Q \cdot \chi=0 \\
& \mathbf{P}\left(q,-\frac{\partial}{\partial q}\right) \chi-\frac{\partial \chi}{\partial Q}=0
\end{aligned}
$$

if they can be derived by these processes. Here $Q(q,-\partial / \partial q)$ or $\mathrm{Q}(q, i)$ denotes $Q$ expressed as a function of $q, p$, and similarly for $\mathrm{P}(q,-\partial / \partial q)$.

We may write these equations analogously to (4), (5), as

$$
\begin{aligned}
& {[\mathbf{Q}(q, p)-Q] \chi=0} \\
& {[\mathbf{P}(q, p)-P] \chi=0 .}
\end{aligned}
$$

It is clear that the passage from $\left(4^{\prime}\right),\left(5^{\prime}\right)$ to $\left(7^{\prime}\right),\left(8^{\prime}\right)$, or from (4), (5) to (7), (8), by the stated processes is exactly equivalent to the algebraic solution of (1), (2) for $Q, P$ in terms of $q, p$, provided that the algebraic operations employed are:

(i) Pre-multiplication by $q, Q, p$ or $P$ or any function of these.

(ii) Permutation of the variables according to the equations

$$
\begin{aligned}
& q Q-Q q=0, \quad q P-P q=0, \\
& Q p-p Q=0, \quad p P-P p=0 \text {, } \\
& q p-p q=1, \quad Q P-P Q=-1 .
\end{aligned}
$$

(iii) Equal quantities may be added or subtracted but not multiplied or divided. 
These are to replace the ordinary rules of algebra for the present set of variables.

Since $q, Q$ commute it does not matter in what order the terms in $W(q, Q)$ and its derivatives are written.

We can solve equation (2), which involves only $p, q$ and $Q$, for the variable $Q$ according to the rules and obtain

$$
\mathrm{Q}(q, p)-Q=0 \text {, }
$$

giving equation (7) at once.

From (10) we obtain, for example,

$$
\mathrm{Q}^{2}(q, p)-Q^{2}=0
$$

where we must obey the rules laid down in forming

$$
\mathrm{Q}^{2}(q, p)=\mathrm{Q}(q, p) \times \mathrm{Q}(q, p),
$$

Proceeding in this way we may write in general, if $F(Q)$ is any function of $Q$,

$$
F\{\mathbf{Q}(q, p)\}-F(Q)=0 .
$$

Consequently if we write (1) in such a way that in each term of $\partial W(q, Q) / \partial Q$ the part depending on $Q$ comes last, we have merely to substitute for $Q$ its value already found in (10). This gives equation $(8)$, and we have now derived (7), (8), or $\left(7^{\prime}\right),\left(8^{\prime}\right)$, in such a manner that $\chi$ is still a solution.

The processes employed may be called pre-multiplication and postsubstitution. It is evident from the operational form of the equations that we may substitute only for a quantity which immediately precedes the $\chi$. In practical cases we may always check the algebra by verifying that $\chi$ remains a solution of any equation obtained. These rules never permit of any ambiguity of interpretation. The only difficulty is that sometimes they do not yield a solution in finite terms of (2) for $Q$. This is the case, for example, if one requires the solution of a general quadratic equation. There seems to be nothing corresponding to the "completion of the square" of ordinary algebra, but we may assume there exists a formal solution in series.

§3. The solution in definite integrals.

Let us now suppose that two functions $\phi(t)$ and $\psi(q)$ are connected by the relation

$$
\psi(q)=\int \chi(q, t) \phi(t) d t
$$

We are going to deal with the two cases where $Q$ satisfies a given equation $(\S 3 \cdot 1)$ and where $\psi$ satisfies a given equation $(\S 3 \cdot 2)$. 
$\S 3 \cdot 1$. We have

$$
\begin{aligned}
& \mathrm{P} \cdot\left(q,-\frac{\partial}{\partial q}\right) \mathrm{Q}^{m}\left(q,-\frac{\partial}{\partial q}\right) \psi(q) \\
& =\int \mathrm{P}^{n}\left(q,-\frac{\partial}{\partial q}\right) \mathrm{Q}^{m}\left(q,-\frac{\partial}{\partial q}\right) \chi(q, t) \phi(t) d t \\
& =\int \mathbf{P}^{\mathbf{n}}\left(q,-\frac{\partial}{\partial q}\right) \chi(q, t) t^{m} \phi(t) d t, \\
& =\int \frac{\partial}{\partial t} \mathbf{P}^{n-1}\left(q,-\frac{\partial}{\partial q}\right) \chi(q, t) t^{m} \phi(t) d t ; \\
& =\left[\mathrm{P}^{n-1} \chi \cdot t^{m} \phi\right]-\int \mathbf{P}^{n-1}\left(q,-\frac{\partial}{\partial q}\right) \chi(q, t) \frac{d}{d t}\left\{t^{m} \phi(t)\right\} d t \\
& =\left[\mathbf{P}^{n-1} \chi \cdot t^{m} \phi-\mathbf{P}^{n-2} \chi \cdot \frac{d}{d t}\left(t^{m} \phi\right)+\ldots+(-)^{n-1} \chi\left(\frac{d}{d t}\right)^{n-1}\left(t^{m} \phi\right)\right] \\
& +(-1)^{n} \int \chi(q, t)\left(\frac{d}{d t}\right)^{n}\left\{t^{m} \phi(t)\right\} d t
\end{aligned}
$$

-on integrating by parts $n$ times.

Suppose now that $\phi(t)$ satisfies a given linear differential equation

$$
G\left(t, \frac{d}{d t}\right) \phi(t)=0
$$

and suppose that the function $G(Q, P)$ can be expanded in terms of the form $P^{n} Q^{m}$. Let each such term be treated as in (12). We obtain then, omitting integrated terms of the type given in square brackets in (12),

$\int \chi(q, t) G\left(t, \frac{d}{d t}\right) \phi(t) d t=G\left\{\mathrm{Q}\left(q,-\frac{d}{d q}\right),-\mathrm{P}\left(q,-\frac{d}{d q}\right)\right\} \psi(q)$.

Now let the path of integration in (11) be so chosen that the sum of these integrated terms vanishes. Then it follows that $\psi(q)$ satisfies the linear differential equation

$$
G\left\{\mathrm{Q}\left(q,-\frac{d}{d q}\right),-\mathrm{P}\left(q,-\frac{d}{d q}\right)\right\} \psi(q)=0
$$

leading to the result:-

If the function $\phi$ satisfies a given differential equation $G(Q, P) \phi=0$, then the definite integral (11), taken along a suitable path, will satisfy the differential equation obtained by substituting for $Q, P$ in $G(Q, P)$ the values of $\mathrm{Q}(q, p)$ and $-\mathrm{P}(q, p)$ derived according to the given rules. The order of the factors in terms like $\mathrm{P}^{n} \mathrm{Q}^{m}$ must be preserved. 
\$3.11. It is to be noted that in deriving (12) we took $n$ to be a positive integer. We shall assume, however, that the definition of differentiation has been so extended that the whole process holds formally also when $n$ is fractional or negative.

\$3.12. Example. We shall use the theorem to prove the known result ${ }^{1}$ that $D_{n}(q)$, the parabolic cylinder function of order $n$, is a constant multiple of

$$
\int_{c} e^{\ddagger q^{2} t}(1+t)^{-\frac{\hbar n}{2}-1}(1-t)^{\frac{1}{2}(n-1)} d t
$$

$C$ is a contour which encircles the point $t=-1$ and begins and ends at $-\infty$.

Here we must evidently take

hence

$$
\begin{aligned}
\chi & =e^{W}=e^{\frac{k 2^{2} t}{4}} ; \\
W & =\frac{1}{4} q^{2} t,
\end{aligned}
$$

giving the appropriate contact transformation.

Equations (1) and (2) become

Solving for $Q, P$ we find

$$
\left.\begin{array}{c}
P=\frac{1}{4} q^{2} \\
p=-\frac{1}{2} q Q .
\end{array}\right\}
$$

$$
\begin{aligned}
& \left.Q=-2 q^{-1} p\right) \\
& P=\frac{1}{4} q^{2}
\end{aligned}
$$

For (16) we must take

$$
\phi(t)=(1+t)^{-\frac{1}{2} n-1}(1-t)^{\frac{n-1}{2}},
$$

which is easily shown to satisfy the differential equation

$$
\left(t^{2}-1\right) \frac{d \phi}{d t}-\left(n+\frac{1}{2}-\frac{3}{2} t\right) \phi=0,
$$

so that in this case

$$
G(Q, P) \equiv\left(Q^{2}-1\right) P-\left(n+\frac{1}{2}-\frac{3}{2} Q\right) .
$$

Calling the expression (16) $\psi(q)$, and using (19) and (22) in (15), we find that it must satisfy the differential equation

$$
\left[\left\{\left(2 q^{-1} \frac{d}{d q}\right)\left(2 q^{-1} \frac{d}{d q}\right)-1\right\}\left(-\frac{1}{4} q^{2}\right)-\left\{n+\frac{1}{2}-\frac{3}{2}\left(2 q^{-1} \frac{d}{d q}\right)\right\}\right] \psi=0,
$$

since the path of integration is seen to have been suitably chosen.

${ }^{1}$ Cf. Whittaker and Watson, Modern Analysis (1927), 353 (Ex. 11) 
This simplifies immediately to

$$
\frac{d^{2} \psi}{d q^{2}}+\left(n+\frac{1}{2}-\frac{1}{4} q^{2}\right) \psi=0
$$

and is the equation for the parabolic cylinder function which is now seen to be satisfied by the integral (16). The integral can then be seen to give the solution which defines this function, when the constant is suitably chosen.

§3·2. Had we solved (1), (2) for $q, p$, obtaining $q=q(Q, P)$, $p=\mathbf{p}(Q, P)$, in place of $\left(7^{\prime}\right),\left(8^{\prime}\right)$ we should have the equations

$$
\begin{aligned}
& \mathrm{q}\left(Q, \frac{\partial}{\partial Q}\right) \chi-q \chi=0 \\
& \mathrm{p}\left(Q, \frac{\partial}{\partial Q}\right) \chi+\frac{\partial \chi}{\partial q}=0
\end{aligned}
$$

We then have from (11)

$$
\begin{aligned}
q \psi(q) & =\int q \chi(q, t) \phi(t) d t \\
& =\int\left\{q\left(t, \frac{\partial}{\partial t}\right) \chi(q, t)\right\} \phi(t) d t,
\end{aligned}
$$

using $\left(7^{\prime \prime}\right)$. If now $\mathrm{q}(t, \partial / \partial t)$ contains a term of the form $(\partial / \hat{c} t)^{n} t^{m}$, the corresponding part of the integral (24) will be

$$
\int\left\{\left(\frac{\partial}{\partial t}\right)^{n} t^{m} \chi\right\} \phi(t) d t=(-1)^{n} \int \chi(q, t) t^{m}\left(\frac{d}{d t}\right)^{n} \phi(t) d t
$$

integrating by parts $n$ times and omitting the integrated part. A similar result holds for $p \psi(q)[=-\partial \psi / \partial q]$, or any function of $q, p$ operating on $\psi(q)$, the $q$ 's and $p$ 's in each term being dealt with in the right order.

Suppose now that $\psi(q)$ satisfies a given linear differential equation

$$
F\left(q,-\frac{d}{d q}\right) \psi=0 \text {. }
$$

Suppose also that the path of integration in (11) has been so chosen that when the substitutions of the form (25) have been made, the sum of the integrated parts vanishes. Then we have the result:-

$A$ given differential equation $F(q, p) \psi=0$ is satisfied by a family of integrals of the form (11), taken along suitable paths, provided the 
function $\phi(t)$ satisfies the differential equation derived from it by substituting for $q$ and $-\partial / \partial q(=p)$ their values in terms of $Q, P$ obtained according to the given rules. The expressions for $q, p$ must be expanded separately in terms of the type $P^{n} Q^{m}$, which must then be written as $t^{m}(-d / d t)^{n}$.

The reversal of the order of the factors in going from $P^{n} Q^{m}$ to $t^{m}(-d / d t)^{n}$ is important, as also is the fact that it must precede the combination of terms arising from successive $q$ 's or $p$ 's.

$\S 3 \cdot 21$. We shall again, as in $\S 3 \cdot 11$, suppose the whole process to hold formally when $n$ is fractional or negative.

$\S 3 \cdot 22$. Example. We shall consider the problem converse to that of $\S 3 \cdot 12$, viz., to find $\phi(t)$ such that

$$
D_{n}(q)=\int e^{t q^{2 t}} \phi(t) d t
$$

We must solve (18) for $q$, $p$, this gives

$$
\left.\begin{array}{l}
q=2 P^{1 / 2} \\
p=-Q P^{1 / 2}
\end{array}\right\}
$$

We start now with the fact that $D_{n}(q)$ satisfies (23) giving

$$
F(q, p) \equiv p^{2}+\left(n+\frac{1}{2}-\frac{1}{4} q^{2}\right)
$$

To obtain the equation for $\phi(t)$ we must substitute $2(-d / d t)^{\frac{1}{2}}$ for $q$, and $(-d / d t)^{\frac{1}{2}} t$ for $p$, using (28) in the general theorem. Hence for $q^{2}$ we substitute

$$
2\left(-\frac{d}{d t}\right)^{1 / 2} 2\left(-\frac{d}{d t}\right)^{1 / 2}=-4 \frac{d}{d t}
$$

and for $p^{2}$ we substitute $\left(-\frac{d}{d t}\right)^{1 / 2} t\left(-\frac{d}{d t}\right) t^{-1 / 2}$.

To reduce the latter we employ Leibniz's Theorem for the derivative of order $1 / 2$, giving

and

$$
\left(\frac{d}{d t}\right)^{1 / 2} t=t\left(\frac{d}{d t}\right)^{1 / 2}+\frac{1}{2}\left(\frac{d}{d t}\right)^{-1 / 2}
$$

$$
\begin{aligned}
\left(-\frac{d}{d t}\right)^{1 / 2} t\left(-\frac{d}{d t}\right)^{1 / 2} t & =-\left\{\left(\frac{d}{d t}\right)^{1 / 2} t^{2}\left(\frac{d}{d t}\right)^{1 / 2}+\frac{1}{2}\left(\frac{d}{d t}\right)^{1 / 2} t\left(\frac{d}{d t}\right)^{-1 / 2}\right\} \\
& =-\left\{t^{2} \frac{d}{d t}+\frac{1}{2} \cdot 2 t+\frac{1}{2} \frac{\left(-\frac{1}{2}\right)}{1.2} \cdot 2\left(\frac{d}{d t}\right)^{-1}\right\} \\
& -\frac{1}{2}\left\{t+\frac{1}{2}\left(\frac{d}{d t}\right)^{-1}\right\} \\
& =\left\{t^{2} \frac{d}{d t}+\frac{3}{2} t^{1} .\right.
\end{aligned}
$$


Substituting (30), (31) in (29) we get for the equation for $\phi$

$$
\left[\left(t^{2}-1\right) \frac{d}{d t}-\left(n+\frac{1}{2}-\frac{3}{2} t\right)\right] \phi=0,
$$

so that $\phi(t)$ is a constant multiple of

$$
(1+t)^{-\frac{1}{2} n-1}(1-t)^{\frac{1}{(n-1)},}
$$

and the path of integration may be taken as in $\S 3 \cdot 12$.

\$4.1. The generalised equations for $\chi$.

If

$$
\left.\begin{array}{l}
Q=h(q, p)\}, \\
P=k(q, p)
\end{array}\right\}
$$

where $h, k$ are given functions, and if when we write $P \equiv \partial / \partial Q, p \equiv-\partial / \partial q$, we obtain a pair of compatible differential equations with the solution $\chi(q, Q)$, then the equations

$$
\left.\begin{array}{l}
Q=\pi^{-1} h(q, p) \sigma \\
P=\pi^{-1} k(q, p) \varpi
\end{array}\right\},
$$

where $\varpi(q, p)$ is any function of $q, p$, also yield compatible differential equations and have the solution $\sigma^{-1} \chi(q, Q)$.

Here $\varpi^{-1}$ is defined by

$$
\varpi^{-1} \varpi=1 \text { or } \varpi^{-1}=1,
$$

which are evidently equivalent.

From the commutation relations $\left(9^{\prime}\right)$ we have

$$
\varpi Q=Q \varpi, \quad \varpi P=P \varpi .
$$

Operating on both sides of (34) with $\varpi(q, p)$, we may therefore write the corresponding differential equations in the form

$$
\begin{aligned}
Q \varpi \chi^{*} & =h_{\varpi \chi^{*}} \\
P_{\varpi} \chi^{*} & =k_{\varpi} \chi^{*} . \\
\chi^{*} & =\varpi^{-1} \chi, \quad \text { these become } \\
Q \chi & =h_{\chi} \\
P \chi & =k_{\chi},
\end{aligned}
$$

which are true by hypothesis, thus proving the Theorem.

In the same way we can show that if the relations

$$
\begin{aligned}
& H(Q, P)=h(q, p) \\
& K(Q, P)=k(q, p)
\end{aligned}
$$


yield compatible equations with the solution $\chi(q, Q)$, then the relations

$$
\begin{aligned}
& \Pi^{-1} H(Q, P) \Pi=\varpi^{-1} h(q, p) \sigma \\
& \Pi^{-1} K(Q, P) \Pi=\varpi^{-1} k(q, p) \varpi,
\end{aligned}
$$

where $\Pi(Q, P)$, $(q, p)$ are any functions of $Q, P$ and $q, p$ respectively, yield compatible equations with the solution $\sigma^{-1} \Pi^{-1} \chi(q, Q)$.

$\S 4 \cdot 2$. The equations $\left(7^{\prime}\right),\left(8^{\prime}\right)$ may be taken as corresponding to (33). But it is now clear that throughout the work we could equally well have taken instead the equations corresponding to (34) derived from $\left(7^{\prime}\right),\left(8^{\prime}\right)$, by means of any arbitrary $\varpi$ function. Or we may replace $\left(7^{\prime \prime}\right),\left(8^{\prime \prime}\right)$ by equations derived from them by means of any arbitrary II function.

It is clear that when $\Pi$ is a simple function of $Q, P$, for example $\Pi=Q$ or $\Pi=P$, the effect of transforming a term such as $P^{n} Q^{m}$ into $\Pi^{-1} P^{n} Q^{m} \Pi$ is, to a greater or less extent, to alter the order of the factors. Thus frequently alteration of the order of the factors will ultimately lead to equations which are compatible and have a common solution, though this will not now be $e^{W}$ but a function usually bearing some resemblance to it. The point is illustrated in the following simple example. Other cases may be observed in Professor Whittaker's paper ( $c f . \S 4,6$ ).

$\S 4 \cdot 21$. Example. Consider the contact transformation

$$
\text { giving } \begin{aligned}
P & =q / Q \\
p & =-\log Q,
\end{aligned}
$$

so that corresponding to $\left(7^{\prime \prime}\right)\left(8^{\prime \prime}\right)$ we have

$$
\left.\begin{array}{l}
q=Q P \\
p=-\log Q .
\end{array}\right\}
$$

remembering the premultiplication rule.

These give compatible equations with the solution

$$
\chi=e^{W}=Q^{q}
$$

Let us choose a $\Pi$ function

$$
\Pi(Q, P)=Q
$$


and form from (37) the relations

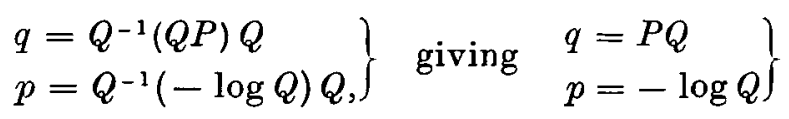

and the corresponding $\chi^{*}$ is

$$
\chi^{*}=Q^{-1} \chi=Q^{q-1} .
$$

If therefore this transformation be employed in an example as in $\S 3.21$ we may employ $\left(37^{\prime}\right)$ and $\left(38^{\prime}\right)$ instead of (37) and (38). This is interesting as an instance where both possible orders of the factors in the solution for $q, p$ lead to consistent equations for a $\chi$ function.

$\S 5 \cdot 1$. An alternative form. The contact transformation need not necessarily be derived from a function of $q, Q$ but may be derived from a function $X(P, p)$ of $P, p$ according to the equations

$$
\left.\begin{array}{c}
Q=\frac{\partial X}{\partial P} \\
q=-\frac{\partial X}{\partial p}
\end{array}\right\} .
$$

In this case we may assert that the pair of differential equations

$$
\begin{gathered}
{\left[Q-X_{p}(P, p)\right] \chi=0} \\
{\left[q+X_{p}(P, p)\right] \chi=0,}
\end{gathered}
$$

where $X_{p}=\partial X / \partial P, \quad X_{p}=\partial X / \partial p$, are compatible and have the solution

$$
\chi(Q, q)=\iint e^{-Q t_{1}-q t_{2}-X\left(-t_{1}, t_{2}\right)} d t_{1} d t_{2},
$$

where suitable paths of integration must be selected. This follows at once from Professor Whittaker's extension of the Laplace Transformation given in $\S 3$ of his paper.

Equations (39) can then be taken in place of (1), (2) as the starting point of the theory, and the appropriate $\chi$-function is given by $(40)$.

It will be immediately evident how to deal with the other possible forms of the contact transformation in which it is derived from a function $Y(q, P)$ of $q, P$, or from a function $Z(Q, p)$ of $Q, p$. The function $\chi$ will then be given as a single integral. 
§5.2. Example. Take $X(P, p)=p P^{2}+\frac{1}{2} \log p$.

Then $\chi(Q, q)=\iint \exp \left(-Q t_{1}-q t_{2}-t_{1}^{2} t_{2}-\frac{1}{2} \log t_{2}\right) d t_{1} d t_{2}$.

Now

$$
\int_{-\infty}^{\infty} \exp \left(-Q t_{1}-t_{1}^{2} t_{2}\right) d t_{1}=\text { const. } t_{2}{ }^{-\frac{1}{2}} \exp \left(\frac{Q^{2}}{4 t_{2}}\right) \text {. }
$$

Assuming for the moment that these limits of integration are allow-able, this gives for $\chi(Q, q)$

$$
\begin{aligned}
\chi(Q, q) & =\text { const. } \int t_{2}^{-1} \exp \left(\frac{Q^{2}}{4 t_{2}}-q t_{2}\right) d t_{2} \\
& =- \text { const. } \int \tau^{-1} \exp \left(\tau-q \frac{Q^{2}}{4 \tau}\right) d \tau \\
& =J_{0}\left(Q q^{\frac{1}{2}}\right),
\end{aligned}
$$

if we may take suitable limits in the last integral. Since now this is. just the $\chi$-function obtained by Professor Whittaker in his $\S 8$ for the analogous contact transformation, we shall assume without further investigation that the limits of integration have been correctly chosen.

We obtain for the contact transformation

$$
\begin{aligned}
Q & =\frac{\partial X}{\partial P}=2 p P \\
q= & -\frac{\partial X}{\partial p}=-P^{2}-\frac{1}{2 p}, \\
\text { or } \quad Q^{-1} P & =\frac{1}{2} p^{-1} \\
P^{2} & =-\frac{1}{2} p^{-1}-q .
\end{aligned}
$$

Let us as a particular case take the $\phi(t)$ of the general theorem to be

$$
\phi(t)=J_{1}(t)
$$

which therefore satisfies the equation

$$
\left[\frac{d^{2}}{d t^{2}}+\frac{d}{d t} \frac{1}{t}+1\right] \phi=0
$$

The equation for $\psi(q)$ then comes to be

$$
\left[\left(\frac{\partial}{\partial q}\right)^{-1}-q+1\right] \psi=0
$$

which shows that $\psi$ is a constant. 
So the result of the general theorem is

$$
\int J_{0}\left(t q^{\frac{1}{2}}\right) J_{1}(t) d t=\mathrm{constant}
$$

for the proper path of integration. ${ }^{1}$

In a somewhat similar way the orthogonal properties of the Bessel functions may be found. But we leave the matter here, intending only to show how in practice the method of this paragraph may allow us to deal with more complicated $\chi$-functions.

$\S 6 \cdot 1$. Successive transformations. Suppose we have

$$
\begin{aligned}
& \psi(q)=\int_{A} \chi(q, t) \phi(t) d t \\
& \phi(t)=\int_{B} \rho(t, Q) \eta(Q) d Q,
\end{aligned}
$$

where $A, B$ denote specified paths of integration. Then

where

$$
\begin{aligned}
\psi(q) & =\int_{A} d t \int_{B} \chi(q, t) \rho(t, Q) \eta(Q) d Q \\
& =\int_{B} \sigma(q, Q) \eta(Q) d Q
\end{aligned}
$$

$$
\sigma(q, Q)=\int_{A} \chi(q, t) \rho(t, Q) d t
$$

We proceed to find the differential equations satisfied by $\sigma(q, Q)$.

The contact transformations yielding the transformations (41), (42), like those of $\S 3$, are derived respectively from the functions

Therefore

$$
W\left(q, q^{\prime}\right)=\log \chi\left(q, q^{\prime}\right) . \quad V\left(q^{\prime}, Q\right)=\log \rho\left(q^{\prime}, Q\right) .
$$

$$
\begin{array}{ccrl}
p^{\prime}=\frac{\partial W}{\partial q^{\prime}} & (46) & P & =\frac{\partial V}{\partial Q} \\
p=-\frac{\partial W}{\partial q} & (47) & p^{\prime}=-\frac{\partial V}{\partial q^{\prime}} .
\end{array}
$$

We first regard (44) as a transformation from $\rho$ to $\sigma$, as functions of $t, q$ respectively, by means of the transformation function $\chi(q, t)$ by

1 This is a particular case of a general result. Cf. Whittaker and Watson, Modern Analysis (1927), p. 385, Ex. 50. It should be noted that

$$
{ }^{1} p^{-1} \chi=Q^{-1} P \chi=Q^{-1} P(q, p) \chi=P(q, p) Q^{-1} \chi=P(q, p) Q^{-1}(q, p) \chi ;
$$

hence the substitution of $\frac{1}{2} p^{-1}$ for $\frac{d}{d t} \frac{1}{t}$ and not $\frac{1}{t} \frac{d}{d t}$. 
the method of $\S 3 \cdot 1$. For by $\left(47^{\prime}\right), \rho(t, Q)$ satisfies the differential equation

$$
\frac{\hat{c} \rho}{\tilde{c} t}-\frac{\partial V(t, Q)}{\partial t} \rho=0,
$$

$Q$ being treated as constant. Then, by the Theorem of $\S 3 \cdot 1, \sigma$ must satisfy the equation

$$
\left[-p^{\prime}\left(q,-\frac{\partial}{\partial q}\right)-V_{t}\left\{q^{\prime}\left(q,-\frac{\partial}{\partial q}\right), Q\right\}\right] \sigma=0,
$$

writing $V_{t}$ for $\partial V / \partial t$. Here $q^{\prime}(q, p), p^{\prime}(q, p)$ are got from (46), (47) by the usual rules of $\S 2$. The partial derivatives denote merely that $Q$ is not affected.

We next regard (44) as a transformation from $\chi$ to $\sigma$ as functions of $t$ and $Q$ respectively by means of the transformation function $\rho(t, Q)$. For by (46), the function $\chi$ satisfies the differential equation

$$
\frac{\partial \chi}{\partial t}-\frac{\partial W(q, t)}{\partial t} \chi=0
$$

$q$ now being treated as constant. Then, by the Theorem of $\S 3 \cdot 1$, $\sigma$ must satisfy the equation

$$
\left[-p^{\prime}\left(Q, \frac{\partial}{\partial Q}\right)-W_{t}\left\{q, q^{\prime}\left(Q, \frac{\partial}{\partial Q}\right)\right\}\right] \sigma=0,
$$

where $q^{\prime}(Q, P), p^{\prime}(Q, P)$ are got by solving $\left(46^{\prime}\right),\left(47^{\prime}\right)$ in the usual manner.

In (49), (51) we have a pair of differential equations for $\sigma(q, Q)$, and we know they are compatible since they are satisfied by $\sigma$ given by (44).

Now if the transformation from $q, p$ to $Q, P$ may be made by a contact transformation specified by a function $U(q, Q)$, we should have

$$
\left.\left.\begin{array}{r}
P=\frac{\partial U}{\partial Q} \\
P=-\frac{\partial U}{\partial q^{-}}
\end{array}\right\} \quad \begin{array}{cc}
P \sigma^{*}=\frac{\partial \sigma^{*}}{\partial Q} \\
\text { or } & p \sigma^{*}=-\frac{\partial \sigma^{*}}{\partial q},
\end{array}\right\}
$$

where $\sigma^{*}=e^{U}$. To find this function $U$ we should solve (46), (47) for $q^{\prime}, p^{\prime}$ in terms of $q, p$ and substitute in $\left(47^{\prime}\right)$, and solve $\left(46^{\prime}\right),\left(47^{\prime}\right)$ for $q^{\prime}, p^{\prime}$ in terms of $Q, P$ and substitute in (46), obtaining in turn

$$
\left.\begin{array}{l}
p^{\prime}(q, p)+V_{t}\left\{q^{\prime}(q, p), Q\right\}=0 \\
p^{\prime}(Q, p)+W_{t}\left\{q, q^{\prime}(Q, P)\right\}=0 .
\end{array}\right\}
$$


If now we convert these into differential equations for $\sigma^{*}$ by writing, according to (52), $P \equiv \partial / \partial Q, p \equiv-\partial / \partial q$, we get precisely equations (49), (51) and so $\sigma^{*}=\sigma$.

The whole elimination leading to equations (53) is of course performed according to the rules of $\S 2$. We have therefore the result:-

In the present non-commutative a!gebra the result of two successive contact transformations specified by the functions $W\left(q, q^{\prime}\right), V\left(q^{\prime}, Q\right)$ is itself a contact transformation specified by the function $U(q, Q)$ given by the integral

$$
e^{U}=\int e^{W(q, t)+V(t, Q)} d t
$$

taken along a suitable path.

We have in this a method for evaluating definite integrals by means of algebraic (non-commutative) elimination.

$\S 6 \cdot 2$. Example. We shall take

Then

$$
W=-q q^{\prime}, \quad V=-q^{\prime 2} Q .
$$

$$
\begin{array}{ll}
p^{\prime}=-q, & P=-q^{\prime 2}, \\
p=q^{\prime}, & p^{\prime}=2 q^{\prime} Q .
\end{array}
$$

It is not necessary to use equations corresponding precisely to (53). Any pair obtained by eliminating $q^{\prime}$ and $p^{\prime}$ suffices. We obtain in fact

$$
\left.\begin{array}{l}
q=-2 Q p \\
P=-p^{2},
\end{array}\right\}
$$

giving as differential equations for $\sigma(q, Q)$

These give readily

$$
\left.\begin{array}{l}
q \sigma=2 Q \frac{\partial \sigma}{\partial q} \\
\frac{\partial \sigma}{\partial Q}=-\frac{\partial^{2} \sigma}{\partial q^{2}} .
\end{array}\right\}
$$

$$
\sigma(q, Q)=Q^{-\frac{1}{2}} e^{q^{2} / 4 Q} \text {. }
$$

On substituting from $(55)$ in $(\$ 4)$ this gives

$$
Q^{-\frac{1}{2}} e^{q / 4 Q}=C \int e^{-q t-Q t^{2}} d t .
$$

It is clear that the appropriate limits of integration are $\pm \infty$, and putting $q=0$ we see that the constant $C$ is $\pi^{-\frac{1}{2}}$. The result is obtainable, of course, by elementary methods. 
§6:3. Example. We shall take

Then

$$
W=(q-1) \log q^{\prime}, \quad V=(Q-1) \log \left(1-q^{\prime}\right) .
$$

whence

$$
\begin{array}{ll}
p^{\prime}=(q-1) / q^{\prime}, & P=\log \left(1-q^{\prime}\right) \\
p=-\log q^{\prime}, & p^{\prime}=(Q-1) /\left(1-q^{\prime}\right),
\end{array}
$$

$$
\begin{aligned}
e^{-p}+e^{P} & =1 \\
(q-1) e^{p} & =(Q-1) e^{-P} .
\end{aligned}
$$

We therefore take as differential equations for $\sigma$

$$
\begin{aligned}
\left\{e \partial \partial \alpha+e^{\partial \partial \partial Q}-1\right\} \sigma & =0 \\
\left\{(q-1) e^{-\partial / \partial \alpha}-(Q-1) e^{-\partial / \partial Q}\right\} \sigma & =0,
\end{aligned}
$$

which are equivalent to the difference equations

$$
\begin{aligned}
\sigma(q+1, Q)+\sigma(q, Q+1) & =\sigma(q, Q) \\
(q-1) \sigma(q-1, Q) & =(Q-1) \sigma(q, Q-1) .
\end{aligned}
$$

The latter are the known difference equations for the Beta function, so we take

$$
\sigma(q, Q)=B(q, Q) .
$$

Substituting from (58) in (54) and adjusting the limits of integration, we get the familiar result

$$
B(q, Q)=\int_{0}^{1} t^{q-1}(1-t)^{Q-1} d t .
$$

$\S 7$. It should be mentioned that a similar theorem to that of the present paper is implicit in some work of P. Jordan on the Quantum Mechanics. ${ }^{1}$ He does not however consider its relation to contact transformations or its pure mathematical implications. On the other hand we have not here considered any possible applications of our methods to the Quantum Mechanics.

$\S 8$. This work was prompted by lectures delivered by Professor E. T. Whittaker on the solution of differential equations by definite integrals, and we wish to thank him for his interest and encouragement.

Is. f. Physik, 40 (1927), 809. 